\title{
Foujita en México: Un episodio de colaboración y mecenazgo, y un retrato de Modigliani
}

\author{
Victoria Soto Caba \\ Universidad Nacional de Educación a Distancia \\ Francisco García Oria \\ Freelance
}

\begin{abstract}
RESUMEN: En 1933 Tsuguharu Foujita viajó a México afincándose en la capital durante casi un año, una estancia que incidió en la evolución formal y temática de su producción artística y que tuvo consecuencias de gran interés para el intercambio artístico y el coleccionismo de la época de entreguerras, ya que sirvió de enlace entre Louis Eychenne, un coleccionista mexicano de la primera mitad del siglo XX, y las obras y artistas de la Escuela de París. El coleccionista le compró obra, le organizó dos exposiciones de dibujos en la capital mexicana que tuvieron cierto éxito y le proporcionaron algunas ventas y Foujita, a cambio, actuó como intermediario de la compra de cuadros, como fue el caso de La Patronne, de Amedeo Modigliani. Se trata de un episodio mexicano poco conocido, como lo son algunas fotografías y dibujos inéditos que se incluyen.
\end{abstract}

PALABRAS CLAVE: Foujita, Coleccionismo mexicano, Louis Eychenne, Modigliani, La Patronne, Escuela de París, Dibujo, Acuarela.

\section{Foujita in Mexico: An Episode of Cooperation and Patronage, and a Portrait by Modigliani}

\begin{abstract}
In 1933 Tsuguharu Foujita traveled to Mexico settling in the capital for almost a year. This stay influenced the formal and thematic evolution of his artistic production with consequences of great interest for artistic exchange and collectors of the between wars period. Also during his stay he served as liaison between Louis Eychenne, a Mexican collector of the first half of the twentieth century, and artists of the School of Paris. The collector bought his work and organized two exhibitions of drawings in Mexico City, provided him with some success and some sales. Foujita, in exchange, acted as intermediary in the purchase of some paintings, as was the case with La Patronne by Amedeo Modigliani. It is a little known Mexican episode. Some unpublished photographs and drawings included.
\end{abstract}

KEY WORDS: Foujita, Mexican Collecting, Louis Eychenne, Modigliani, La Patronne, École de Paris, Drawings, Watercolors.

Recibido: 2 de abril de 2013 / Aceptado: 2 de mayo de 2013

Tsuguharu Foujita fue uno de los artistas japoneses más viajeros de la primera mitad del siglo $X X$, con una trayectoria inmersa en las fluidas relaciones que, entre Europa y el resto de los continentes, se dieron en el periodo de entreguerras, así como un reflejo del abandono de la política de aislamiento que sufrió Japón

* SOTO CABA, Victoria y GARCÍA ORIA, Francisco: «Foujita en México: Un episodio de colaboración y mecenazgo, y un retrato de Modigliani", Boletín de Arte, $n .{ }^{\circ} 34$, Departamento de Historia del Arte, Universidad de Málaga, 2013, pp. 297-317, ISSN: 0211-8483. 
y, en consecuencia, de la apertura a Occidente durante el periodo Meiji. Nacido en Tokio en 1886, ciudad en la que estudió, formándose en la Escuela de Bellas Artes, se trasladó a París en 1913, en un primer viaje a la capital gala en donde coincidió y entabló amistad con pintores de las más diversas nacionalidades, como Modigliani, Rivera o Soutine, entre otros muchos ${ }^{1}$. Tras este viaje, casi de reconocimiento, Foujita acabará instalándose en 1915 para pasar a formar parte del cosmopolita y heterogéneo grupo de artistas que se conoce como Escuela de París ${ }^{2}$, dentro de un contexto estilístico relacionado con la creación figurativa que un buen número de pintores produjeron durante las primeras décadas del siglo XX. Como Chagall o Kisling, representa la simbiosis entre el estilo o los rasgos propios de los países de origen y las nuevas propuestas plásticas europeas. En Foujita, la seducción que produce su amplia obra, de óleos y dibujos, decoraciones murales e ilustraciones para libros, reside precisamente en esa síntesis entre las formas orientales y las fórmulas de la vanguardia figurativa, consiguiendo un estilo propio que unificó las técnicas japonesas tradicionales y la pintura al óleo tradicional, con una postura que ha sido calificada «entre Oriente y Occidente ${ }^{3}$, una perspectiva que proporciona a sus composiciones una imagen extrañamente moderna, de un refinamiento extremado $y$, en el caso de sus desnudos femeninos, de un erotismo discreto y a la vez sofisticado.

Durante la década de los años veinte el pintor se integró en los círculos de la vida bohemia parisina, adquiriendo una alta notoriedad, por la seducción de sus nacarados desnudos, perfilados sobre masas blancas, y la originalidad de sus retratos y naturalezas muertas, por su interpretación de los asuntos occidentales que no conseguían borrar su distintivo oriental, un dualismo y una fusión a la vez de elementos antitéticos que le abrió las puertas del escaparate artístico de la capital gala, tal y como se recogía en la prensa de la época ${ }^{4}$. Pero también su

1 Tal y como reflejó la pintora rusa Marevna Verobev-Stebelska en un dibujo firmado y fechado en 1913, en el que se recoge una visita a La Ruche indicando los nombres de los artistas referidos y el de Foujita, dibujo publicado por BUISSON, Sylvie, Foujita et ses amis du Montparnasse, París, Éditions Alternatives, 2010, p. 53. No obstante, toda la vida en París y la relación con sus amistades las documentó Foujita constantemente en sus dibujos y su producción pictórica.

2 A pesar de la discusión y el rechazo que viene provocando desde hace tiempo la agrupación de esta "escuela" y su denominación como tal, son todavía muchos los trabajos que continúan insistiendo en este término, véase por ejemplo el catálogo de la exposición L'École de Paris, 1904-1929, la part de I'Autre, celebrada en el Musée d'Art Moderne de la Ville de París, del año 2000.

3 Así se califica en una de las últimas biografías críticas del artista, cfr. BIRNBAUM, Phyllips, Glory in a Line: A life of Foujita. The artista caught between East and West, Nueva York, Faber \& Faber, 2006. Con anterioridad, la convergencia entre Oriente y Occidente en la obra del artista japonés ha sido patente en la exposición comisariada por BUYSSON, Silvie en el Centre Cultural Bancaixa de Valencia; cfr. el catálogo respectivo Foujita, entre Oriente y Occidente, Valencia, 2005.

4 Véase GOMEZ DE LA MATA, Germán, «El japonés Foujita», Nuevo Mundo, 18 de febrero de 1927, n. 1726. 
reputación venía dada por su extravagante y caprichosa personalidad, así como por su inestable vida sentimental. Divorciado sucesivamente, de Tomiko, su mujer japonesa al llegar a París, de Fernande Barrey, con quien se casó en 1917, y de Lucie Badoul, Youki, su tercera esposa, a la que conoció en 1921, tendría en Madelaine Lequeu a una de sus últimas amigas ${ }^{5}$. Con esta última emprendió en 1931 un largo viaje por América Latina, un periplo que le llevaría a recorrer Brasil, Argentina, Bolivia, Perú, Cuba, Panamá, México y el sudoeste de los Estados Unidos, y que terminaría en Japón a finales de 1933. Aunque con alguna visita esporádica y puntual, como la de 1929 para estar presente en una gran exposición que se le brindó en Tokio y Osaka, Foujita llevaba más de diecisiete años ausente de su país natal.

Los motivos del viaje a América han sido explicados de diverso modo. Sylvie Buisson argumenta una huida hacia delante tras la ruptura del artista con Youki, máximo cuando la Badoul se convirtió en amante de un gran amigo del nipón, el poeta surrealista Robert Desnos. No obstante, viajó acompañado de la citada Madelaine, la controvertida modelo adicta a la morfina. El anhelo de conocer mundo pudo ser otro motivo antes del regreso a su país, así como el reclamo familiar reiterado; pero Foujita, que había conseguido sustanciosas ventas y exposiciones de resonado éxito, especialmente en la de 1928, celebrada en la Galería Bernheim-Jeune, llegando a ser uno de los artistas más ricos del grupo de París, con reconocimientos oficiales, como la concesión de la Legión de Honor francesa en 1925, se encontraba completamente arruinado tras unos años de vida lujosa y continuos derroches. Deseaba volver como un triunfador y para ello dejó encargado a Desnos la venta de todas sus pertenencias, y planeó una producción de obra que llevaría allende los mares con vistas a recaudar el dinero suficiente para retornar a su país. Era un artista extremadamente cultivado y perfectamente consciente de que ocupaba una posición singular en París ${ }^{6}$. Se pertrechó para el viaje de dibujos de gatos y desnudos femeninos que tanto habían gustado al público parisino y serían bienvenidos por los coleccionistas americanos, temas que copiaría y multiplicaría durante todo el periplo, abordando de forma reiterada estos asuntos destinados a la venta, en una actitud que podría calificarse de avida dolars ${ }^{7}$. Por otro lado, no se descarta que Foujita

5 Casi todas ellas, figuras del ambiente alegre y distendido del París del primer tercio del siglo XX, son tratadas en el ilustrado trabajo de KLÜVER, Billy y MARTIN, Julie, El Paris de Kiki. Artistas y amantes 1900-1930, Barcelona, Tusquets, 1990.

6 SILVER, Kenneth E., "Made in Paris", L'École de Paris, 1904-1929, la part de l'Autre, ob. cit., p. 42.

7 Una actitud que no pasó desapercibida para algunos críticos. En un comentario de la prensa española de 1933, palpitante de racismo, un autor con el pseudónimo de Amichatis se refería al interés 
llevara también trabajos de sus colegas pintores, dibujos, cartones y algún lienzo de sus amigos parisinos, como Modigliani o Picasso -el primero ya fallecido, pero reverenciado desde la aparición de la biografía de André Salmon en 1926 y de las celebraciones de las Bienales de Venecia de 1922 o de 1930, y el segundo completamente asentado en los circuitos artísticos y comerciales-, y con quienes había tenido costumbre de intercambiar obras, obras que además podían provenir del acopio de piezas que poseían algunos mecenas, galeristas y coleccionistas del París de aquellos años, como Guillaume o Zborowski, todavía vivos, muy cercanos al artista japonés, cuyo viaje serviría de vehículo comercial; se convertía, pues, en un intermediario con un material que sin duda alguna tendría una rotunda salida mercantil en el ámbito americano.

Pero, frente a las razones crematísticas del viaje, Foujita tenía un vivo interés por visitar América, especialmente México. En una entrevista que le realizaron en 1930, tras las exposiciones en Tokio y Osaka, le preguntaron si había vendido muchos cuadros en Estados Unidos, pues de regreso tuvo que arribar en Nueva York y San Francisco; el artista contestó: "Los Estados Unidos no me interesan. Ex profeso no he querido vender casi nada en ese país. En cambio, México es una tierra que me encanta, y adonde iría de buena gana. Conocí un mexicano, un gran pintor, se llama Diego Rivera» ${ }^{8}$.

Por el cono sur Foujita inició una renovación temática durante sus casi tres años de desplazamientos, elaborando una producción nueva de dibujos, acuarelas y óleos que se ofrecieron al público sudamericano con la celebración de varias exposiciones, dibujos algunos inéditos y exposiciones desconocidas e ignoradas en los catálogos sobre el artista. Los estudiosos no acaban de ponerse de acuerdo con las etapas y escalas del viaje; sin duda se iniciaron en Brasil, para continuar en Argentina, desde marzo de $1932^{9}$ hasta julio del mismo año, donde celebró una exposición de enorme éxito, para seguir por Bolivia y Perú, de agosto a octubre, y en este mismo mes recalar en los países caribeños, en concreto en Cuba, donde expuso con notable repercusión en el Lyceum de la Habana durante el mes de noviembre ${ }^{10}$. A finales de 1932 viaja a México, aunque

pecuniario del japonés en los siguientes términos: "¿afán artístico? No... Asia está orgullosa de su tradición artística y desprecia la escuela europea. Foujita, de París, solo ha aceptado dinero", El Heraldo de Madrid, 30/1/1933, p. 16.

8 La Esfera, 31 de mayo de 1930, año XVII, n. ${ }^{\circ} 856$, p. 44.

9 Una revista bonaerense asegura que el 28 de marzo "llegó el famoso pintor japonés Foujita" en Caras y Caretas, 9/4/1932, n. ${ }^{\circ} 1749$, p. 98.

10 Para la repercusión en la prensa cubana sobre la exposición y la estancia de Foujita en Cuba véase Opus Habana, n. ${ }^{\circ}$ 2, 2003, pp. 40-47 en <http://www.opushabana.cu/index.php?option=com_content\&ta sk=view\&id=276\&ltemid=43> [16/01/2013]. 


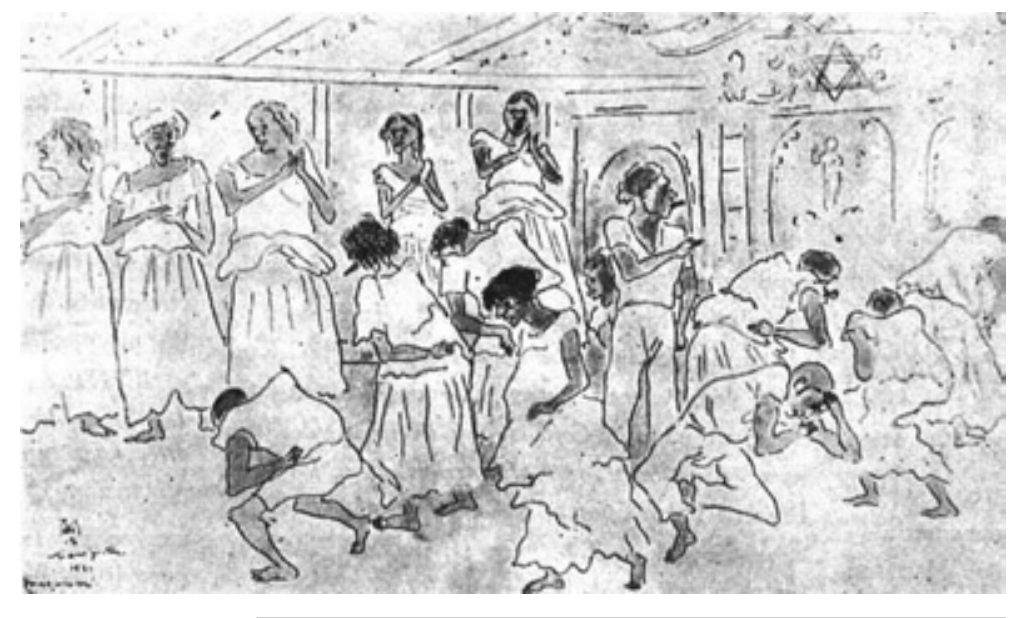

1. La Macumba. Dibujo reproducido en la revista Monterrey. Correo Literario de Alfonso Reyes. Río de Janeiro, marzo de 1932, n. ${ }^{\circ}$ 8, p. 1

11 Monterrey. Correo Literario de Alfonso Reyes, Río de Janeiro, marzo de 1932, n. ${ }^{\circ}$ 8, p. 1. 


\section{E artículos Victoria Soto Caba y Francisco García Oria}

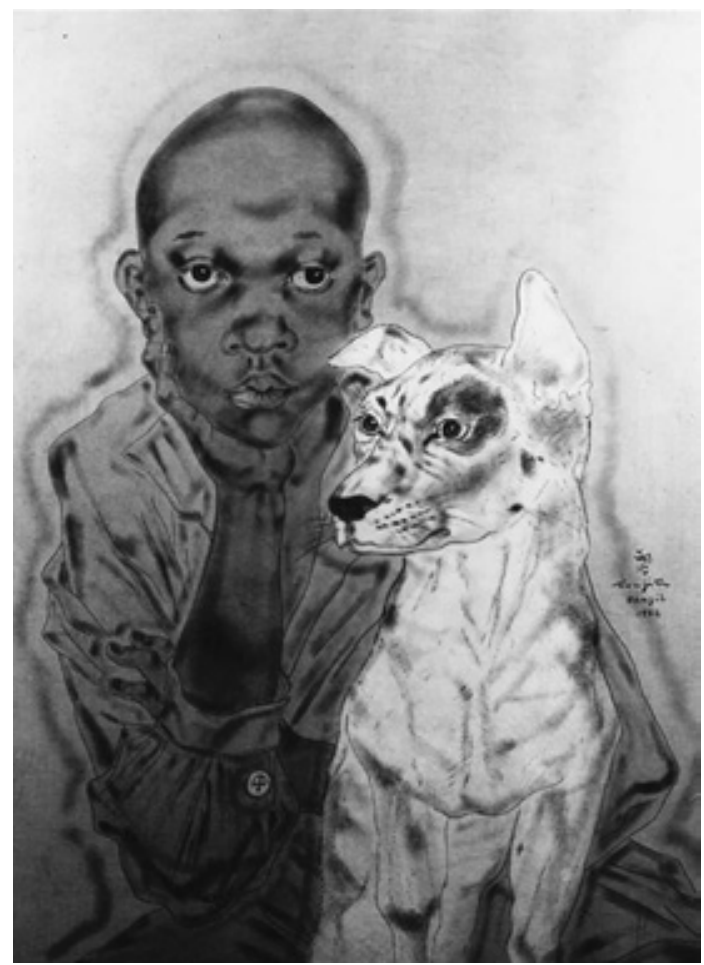

2. Joven mulato con perro, 1932. Colección Louis Eychenne, México D.F.

en la capital y solitario en sus campos y pueblos, como Foujita reflejó en otra acuarela de 1933, Fonda y café Toda Hora [4], de una quietud, una serenidad y una soledad parangonable a los ambientes y escenarios de la pintura metafísica, pero que en realidad continúan el gusto que tuvo en París por captar rincones y esquinas del paisaje urbano, los rincones enternecedores de los arrabales, de la banlieue, impregnados de una apariencia reveladora, misteriosa y mágica, muy alejada de lo cotidiano. No obstante, es evidente que en América su estilo ha evolucionado, ha ganado en contrastes y en riqueza cromática, sustituyendo el blanco nacarado de sus anteriores figuras, que prescinden del color, por tonalidades ocres y rojizas, tonos siempre contenidos, pues nunca fue un pintor colorista en el sentido occidental.

Lógicamente, mantuvo sus iconos, sus emblemas y referencias, los personajes de su refinado universo y la iconografía más demandada por el público; sus fetiches en suma, como gatos y perros, que se datan desde su llegada, como el Chat endormi [5], una acuarela y tinta china de 1932, un ejemplo de entretan- 


SE artículos $\quad$ Foujita en Mexico...

3. Familia mexicana, 1933. Colección Louis Eychenne, México D.F.

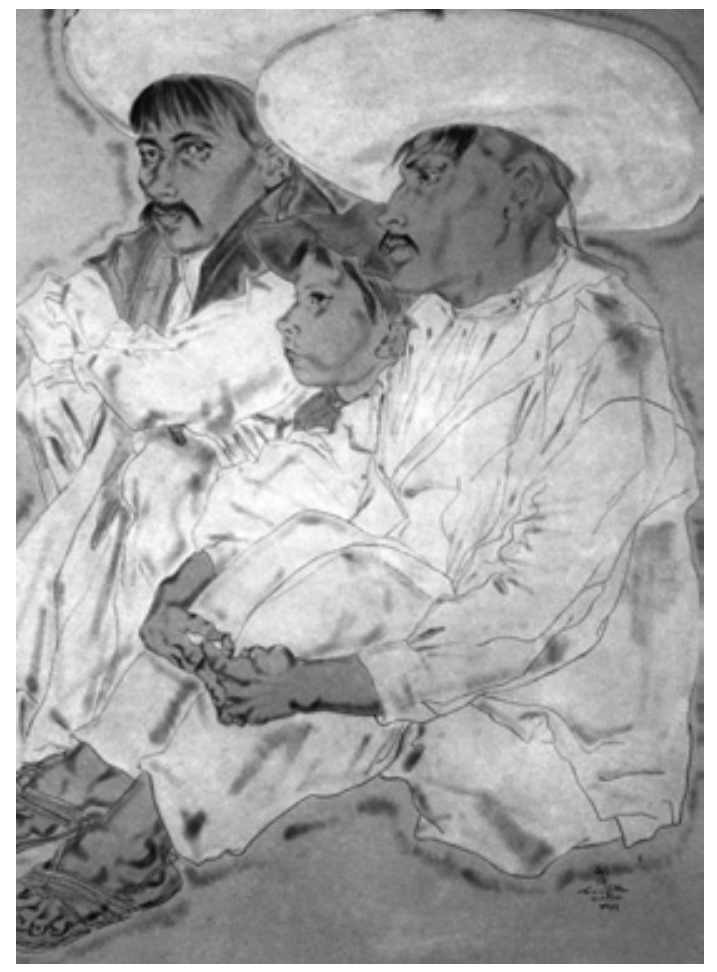

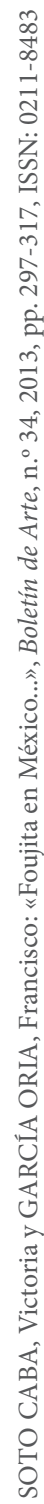




\section{- artículos Victoria Soto Caba y Francisco García Oria}

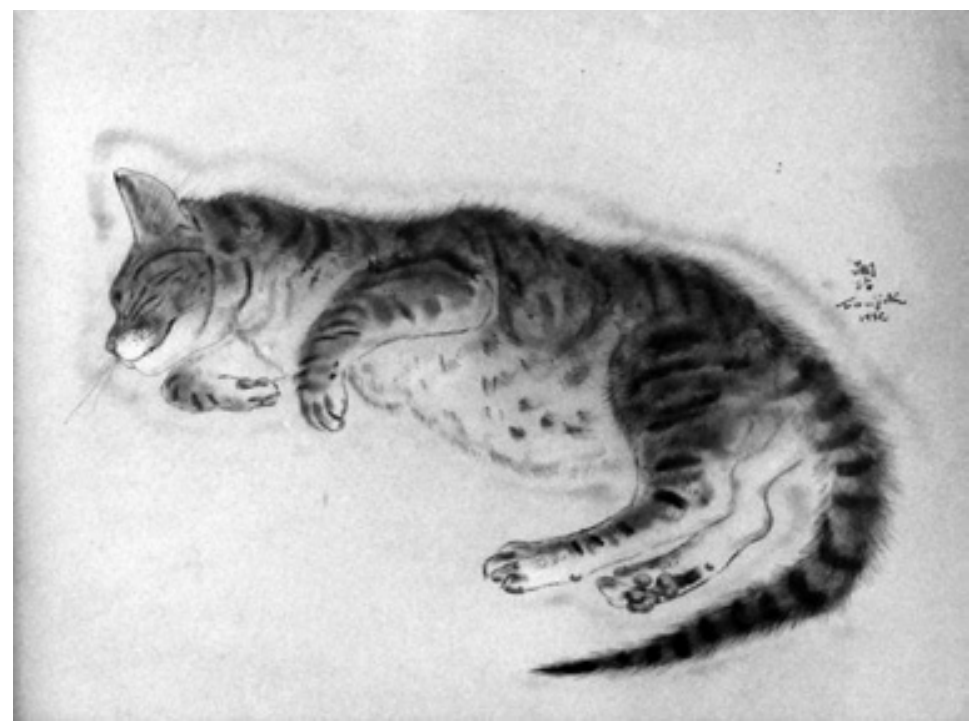

5. Chat endormi, 1932. Colección Louis Eychenne, México D.F.

tos y que reiteró en distintas versiones, en unos casos en distintas posturas, en otros en copias miméticas destinadas a la venta ${ }^{12}$.

Muy pocas noticias, por no asegurar que ninguna, encontramos de las condiciones del viaje que la pareja, Foujita y Madelaine, tuvieron que soportar en los años treinta al trasladarse de un país a otro, de una ciudad a otra. Además del camarote del barco que les llevó a América y de los hoteles, la pareja fue alojada por amigos y contactos que el japonés ya había entablado y organizado desde París. Alojamientos que dieron un respiro al pintor y le permitieron seguir cultivando uno de sus géneros predilectos, el desnudo, siendo Madelaine el modelo exclusivo durante estos años, mostrada desnuda, durmiendo y resaltando su rizada melena, autorretratándose con ella, y en ocasiones con un gato, dibujos que reflejan claramente las fotografías que, desde 1931, se había realizado con Mad y que revalida la utilización del soporte fotográfico en su proceso creativo, y en el cual sigue desarrollando su peculiar estilo personal, basado en la línea como cualidad superior al volumen y la forma. En estos dibujos y acuarelas los relieves son secundarios, se simplifican ante la continua estilización y el arabesco, donde el pincel funciona como una pluma e, incluso, como un buril, siempre

12 Un Chaton endormi, de 1927, muy similar al señalado lo vendió en Buenos Aires en 1932 -actualmente se oferta en Christie's-. Podría decirse que dispersó por Hispanoamérica un buen número de acuarelas y dibujos a tinta china dedicadas a perros y gatos. 
meticuloso y ordenado. Como buen oriental, la tinta china era su punto fuerte, su trazo y su línea eran esencialmente japonesas ${ }^{13}$, la expresión estilística de su diferencia cultural, de su identidad. También sigue interesado en una paleta de tonos claros, de tonos pasteles, aplicada en capas ligeras, finas y delicadas, que otorgan al cuadro una sensación de planicie, de volúmenes planos rodeados de un trazo negro y sinuoso.

Una parte de las obras dedicadas a diversos personajes y tipos que el pintor encontraba en las ciudades y los campos de Sudamérica, en muchos casos inéditas, así como otras obras características del universo plástico de Foujita, acabó siendo adquirida por Louis Eychenne, un apasionado coleccionista mexicano, poco conocido por su celosa discreción pública ${ }^{14}$, una figura con quien el pintor inició una larga amistad. Se conocieron a comienzos de 1933, recién llegado a México. La amistad durará mucho tiempo y en ella la colaboración tuvo un papel destacado. Foujita le retrató en esa misma fecha, Retrato de Louis Eychenne [6] y el mexicano, en el casi un año que estuvo con el japonés, se convirtió en su cicerone y mecenas.

Louis Eychenne había nacido a finales del siglo XIX en Francia, en una localidad de la Provenza, y emigrado a México en plena Gran Guerra. En pocos años se hizo con una enorme fortuna y pasó a formar parte de la élite social y económica del México postrevolucionario de Vasconcelos. $Y$ es en este contexto donde se inicia como coleccionista, adquiriendo entre 1920 y 1934 una gran cantidad de obras de artistas contemporáneos mexicanos, amigos personales como David Alfaro Siqueiros, Diego Rivera y Frida Khalo, así como pintura antigua que compra a colecciones privadas de poderosas familias mexicanas. En 1932 aparece ya como prestador de obra mexicana para algunas exposiciones en Estados Unidos. Sin embargo, llevaba varios años intentando ampliar su colección con unos planteamientos más renovadores, más modernos, más internacionales. Estaba interesado especialmente por los artistas europeos de vanguardia. Cuando alguno de estos artistas viajaba a México, Louis procuraba entablar amistad a la par que ejercer de cliente o marchante. Dentro de esta política interesada y perso-

13 Cfr. LE LIBERDER, Anne et al.: Foujita: le maître du trait, París, Philippe Picquier, 2008.

14 El coleccionismo durante el primer tercio del siglo XX tuvo en México una gran expansión, no comparable a la estadounidense, pero de enorme interés; sin embargo la figura de Louis Eychenne como coleccionista no aparece en el documentado libro de GARDUÑO, Ana, El poder del coleccionismo de arte: Alvar Carrillo Gil, México, UNAM, 2009. El nombre de este coleccionista sí aparece en catálogos de exposiciones para las que prestaba obra; la única aproximación a esta figura en GARCÍA ORIA, Francisco y SOTO CABA, Victoria, "Entre Francia y México: la colección de Louis Eychenne» en ANTIGÜEDAD

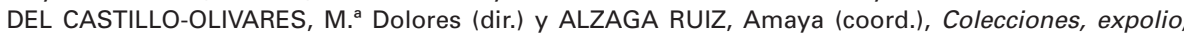
museo y mercado artístico en España en los siglos XVIII y XIX, Madrid, Editorial Universitaria Ramón Areces, 2011, pp. 353-380. 


\section{E artículos Victoria Soto Caba y Francisco García Oria}

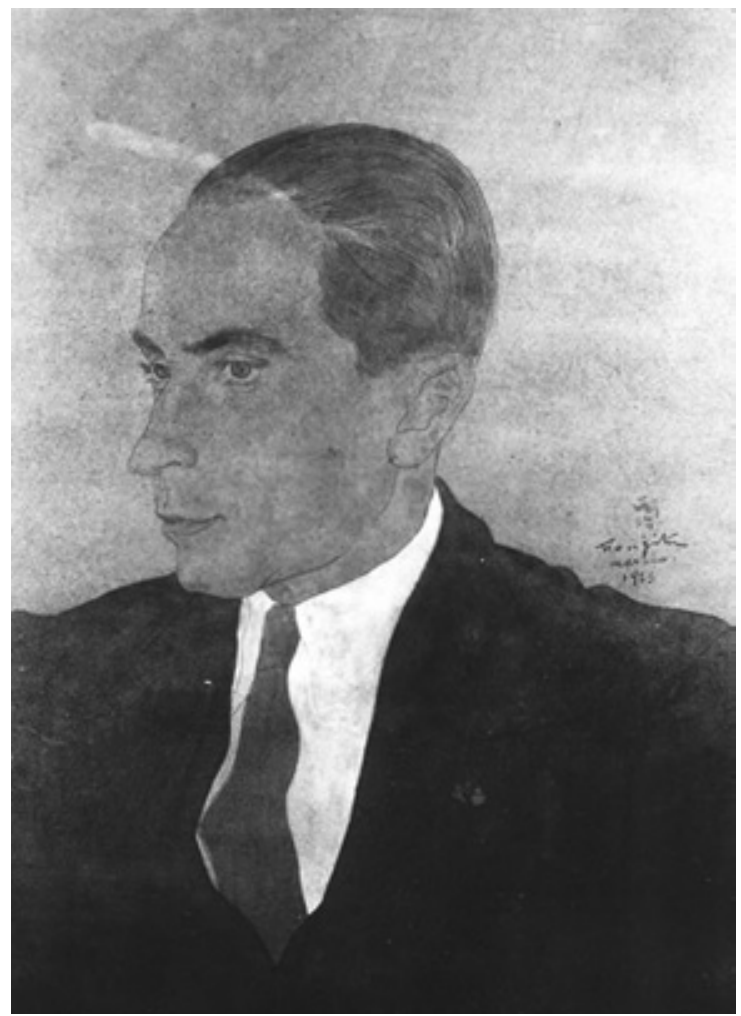

6. T. Foujita: Retrato de Louis Eychenne, 1933. Colección Louis Eychenne, México D.F.

nal, en 1932 invitó a conocer México a Jacques Lipchitz y su esposa, y gracias a ellos parece ser que conoció a Foujita y Madelaine, aunque también es probable que la presentación la realizara Siqueiros. Louis estaba altamente interesado en contactar con el japonés, conocía perfectamente quien era y su éxito en Europa, sabía de su cotización y de las críticas internacionales ${ }^{15}$.

Eychenne se convirtió en el cicerone de la pareja durante el periodo que Foujita y Madelaine viajaron al interior del país, visitando diversas localidades. Se fotografiaron casi siempre, y en muchas de estas fotografías, firmadas y dedicadas por Foujita y Madelaine [7, 10 y 11], se refleja no solo la gran amistad que mantuvieron, sino la admiración de la francesa por el mexicano. Louis se sintió fascinado por Mad y cuando ella regresó a París mantuvo una muy perso-

15 Un dato certero es que en el archivo privado del coleccionista se encuentra el número monográfico que la revista L'art et les artistes dedicó exclusivamente al artista japonés, de octubre (n. $\left.{ }^{\circ} 120\right), 1931$. 


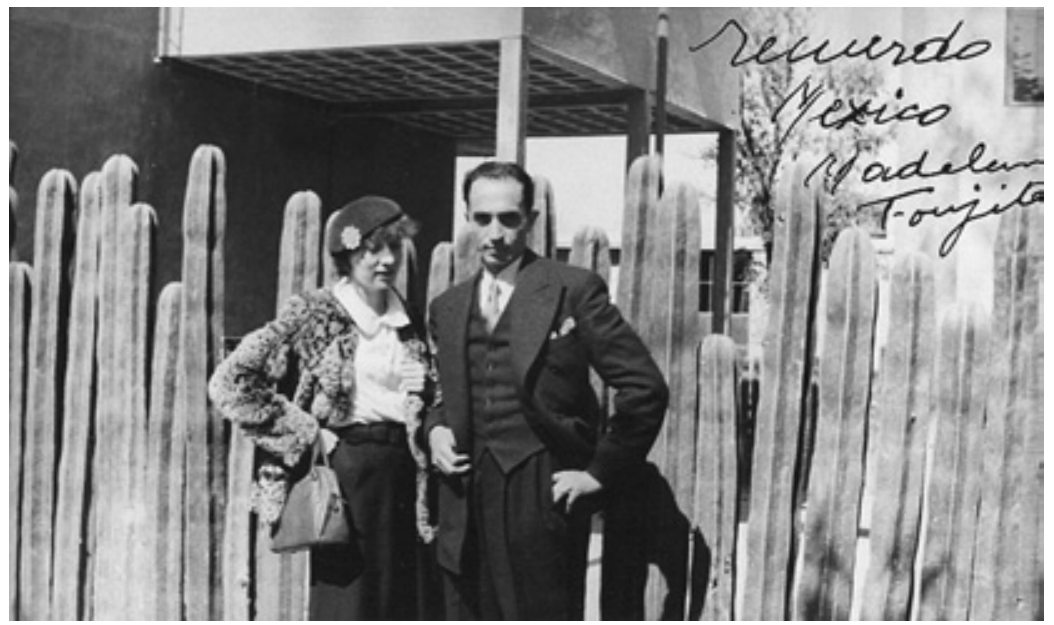

7. Madelaine Lequeu y Louis Eychenne en la Hacienda de Los Morales, México. Fotografía dedicada, tomada por T. Foujita, en 1933. Colección Louis Eychenne, México D.F.

nal correspondencia ${ }^{16}$. Además de cicerone, Eychenne operó como mecenas al organizar exposiciones de dibujos de Foujita en la capital, invitando a su círculo de amistades y gracias a las cuales el pintor consiguió ventas, ingresos y un éxito reconocido, especialmente en la primera, exclusivamente dedicada a temas mexicanos, celebrada en el edificio de La Nacional del 12 al 17 de junio de $1933^{17}$.

Sin embargo, al coleccionista mexicano le interesaba la amistad con Foujita por otros motivos, que no son otros que el de la relación y el enlace que el japonés podía proporcionar con otros pintores afincados en París. Se ha mencionado anteriormente la posibilidad de que el artista viajara a América con obra de pintores amigos suyos con el fin de venderla y conseguir dinero, viajaba también con contactos para organizar su visita a México, como los que le pudieron ofrecer Diego Rivera o Siqueiros, con quienes coincidió en París -de hecho, una de las primeras visitas que realizó el japonés en México fue al estudio de Siqueiros-, dos artistas por otro lado muy ligados a Eychenne, pues este les compró obra y además fue retratado por Siqueiros. Foujita era el enlace seguro con otros artistas cuya obra anhelaba, especialmente de Modigliani o Picasso. El japonés

16 Se conservan las cartas que Madelaine Lequeu escribió a Louis Eychenne en el archivo de los herederos del coleccionista.

17 Se expusieron 40 dibujos como se detalla en un folleto de dos hojas publicado para la ocasión: Exposición de dibujos con temas mexicanos por Foujita. 12 al 17 de junio de 1933. Entre las 16 y las 20 horas. Central de Publicaciones, S.A. Edificio "La Nacional». México D.F. 
se convirtió en una especie de proveedor y de consejero, como una pieza más en el complejo juego de interrelaciones sociales entre artistas, clientes, críticos y coleccionistas, el grupo más influyente en las valoraciones artísticas de la vida cultural de la época de las vanguardias. De esta forma, el mexicano coleccionó no solo obras del japonés ${ }^{18}$, sino que consiguió cuadros europeos, los de la denominada Escuela de París, además de otros artífices franceses de las tres primeras décadas de siglo. Así, pues, a través de Foujita y entre los años de 1933 y 1935 entraron en la colección Eychenne obras como un Retrato de Yvette de Toulouse-Lautrec, una Coronación de espinas de Georges Rouault, unos Saltimbanquis de Picasso y el retrato de una joven, La Patronne de Modigliani, entre otros. Muchos de estos cuadros, así como las obras de Foujita, le sirvieron al mexicano para establecer una relación de intercambio con otros coleccionistas y marchantes, tanto en México como en Estados Unidos, pero de las obras citadas nunca se desprendió.

La Patronne fue su pieza favorita [8]. Eychenne era perfectamente conocedor de la repercusión y de la importancia de Modigliani, pues apenas tres años antes, en la Bienal de Venecia, en la que se expuso un buen repertorio de retratos, las ventas de modiglianis se incrementaron para nutrir los mejores museos del momento y las más exclusivas colecciones privadas. Por otro lado, no hay que olvidar que Modigliani había coincidido en París con varios pintores muralistas que, décadas después, en los años treinta serían amigos de Louis Eychenne, como ya se ha mencionado, los citados Rivera y Siqueiros. Varios bocetos de Modigliani, óleos sobre cartones, de 1914, y un lienzo de la misma fecha, recogen la cara oronda y la rotundidez física de Rivera, cuando se conocieron en La Rotonde, un día que estaba acompañado por León Trotsky. El muralista acabó alojando al pintor durante algunos meses en su casa, en el n. ${ }^{\circ} 16$ de la rue Saint Gothard ${ }^{19}$. Allí realizó esos retratos. Es fácil suponer, que los pintores mexicanos contaron al coleccionista numerosas historias sobre el pintor italiano.

La Patronne es un cuadro que se ajusta a las dimensiones características de Modigliani, pero se trata de un óleo sobre cartón, un soporte poco habitual

18 Entre las obras de Foujita que forman parte de la colección Eychenne, dibujos, acuarelas y óleos en papel, cartón y lienzo, materiales y técnicas que en numerosas ocasiones unificó, destacan, además de las indicados en el texto, una serie de retratos: Autorretrato con Mad y un gato, Madelaine durmiendo, Madelaine desnuda, las tres firmadas y fechadas en 1932; Perro jugando y Mujer cuzqueña con niño a la espalda, también de 1932; y la serie mexicana de 1933: Viejo campesino mexicano, Pareja mexicana, Dos niños, Niño mexicano y Familia mexicana. Nunca se han reproducido ni han formado parte de ninguna exposición, excepto de la mexicana de 1933 en donde las adquirió Eychenne. Tampoco se incluyen en el exhaustivo trabajo de BUISSON, Sylvie, Léonard Tsuguharu Foujita. Catalogue de l'oeuvre, 2 vols. Paris, ACR edition, 2001.

19 SOTO CABA, Victoria, Modigliani, el rostro intemporal, Madrid, Libsa editorial, 2008, p. 262. 
8. La Patronne de Amadeo Modigliani, c. 1916-1919. Óleo sobre cartón, 65 x $45 \mathrm{~cm}$. Colección Louis Eychenne, México D.F.

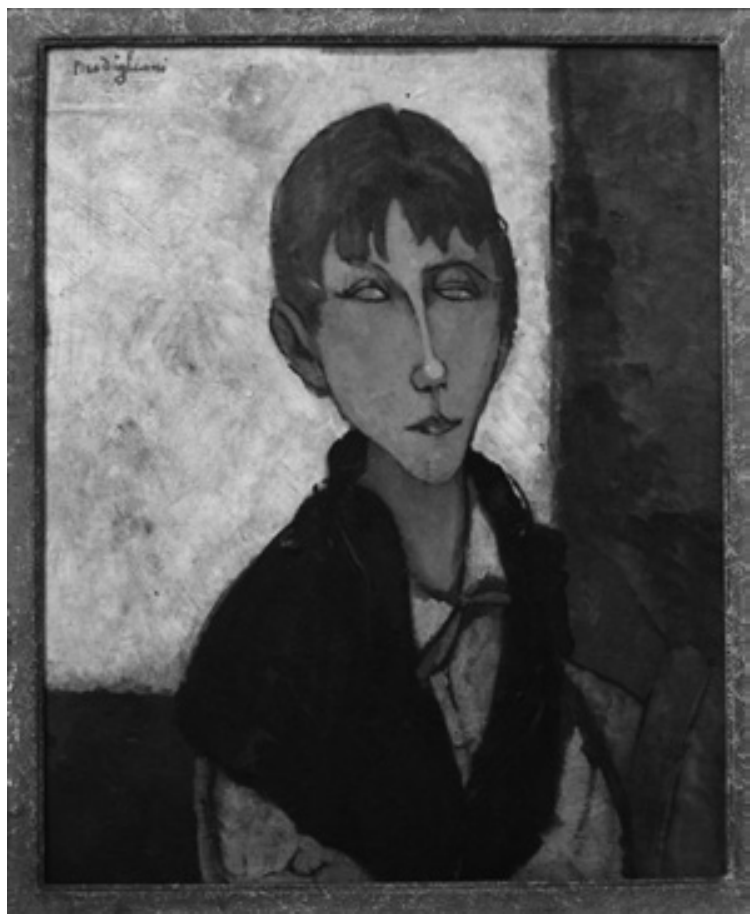

en los cuadros del artista italiano, a excepción de bocetos preparatorios y explicables en unas fechas coincidentes con la carestía de la guerra. Por los rasgos del retrato y el estilo, superada la etapa primitivista y escultórica (1911-1913), así como las geometrizaciones de los años 1914-1916 (Madam Pompadour, L'enfant gras, Los esposos), se ha datado el cuadro en torno a los años de 1917-1918, años en los que configura un modelo retratístico de síntesis, con un estilo propio, muy lineal, estilizado, formalmente distorsionado y ajeno a la perspectiva, cuya composición se divide por una fuerte vertical que obliga a diferenciar la abstracción del plano y el fondo, para sugerir una pared, un vano o una puerta, una solución que llevaba ensayando desde sus comienzos para amortiguar sus fondos neutros. En el caso de La Patronne la división claramente rectangular apunta a una pared blanca, a un vano o a una ventana, y añade además un elemento del mobiliario, la parte superior de un asiento, de una silla, que será una incorporación palpable desde 1916, y una característica de sus últimos retratos, en especial toda la serie que realiza a su compañera Jeanne Hébuterne en 1918, o la de los muchachos sentados del mismo año. La composición ha evolucio- 
nado con respecto al encuadre característico de los primeros momentos, desde que lo propuso esquemáticamente en La cabeza roja, en 1915, una fórmula cezanniana por la cual a partir de la intersección de dos ejes, coincidentes con las verticales y horizontales del fondo, se marca el centro de la cabeza. Esta forma de encuadrar tanto las cabezas, como los rostros y óvalos fue atemperándose al desplazar lateralmente las verticales que confeccionan los fondos y La Patronne es ya un ejemplo de ello.

La compra o el intercambio del cuadro debieron formalizarse en el verano de 1933, llegando el cuadro a la residencia de Eychenne en los últimos meses de ese año, o probablemente a comienzos de 1934, año en que el coleccionista se casa con Dolores Cuevas $^{20}$, aunque la incógnita de cómo se compró y como llegó a México está todavía por esclarecer ${ }^{21}$. Hay una serie de hechos que deben manejarse para explicar ciertas hipótesis del traslado de este cuadro y su Ilegada. Dado que resulta difícil imaginar que Foujita viajara con la obra durante aquel periplo por Sudamérica hasta llegar a México, sí que hay considerar, sin embargo, los continuos viajes del coleccionista Eychenne a Francia, especialmente a partir de 1932, fecha en la que empieza a enviar parte de sus cuadros a su localidad natal para reagrupar allí una colección de pintura española y mexicana, tramitando desde 1933 permisos de exportación con puerta de salida en Veracruz ${ }^{22}$. Por lo que también es probable que fuera Eychenne el encargado de recoger la obra a Francia tras la compra.

20 Perteneciente a una de las poderosas familias hacendadas mexicanas desde el siglo XIX, heredera de una nutrida colección de obra antigua y pintura novohispana; véase GARCÍA ORIA, F. y SOTO CABA, V., ob. cit., pp. 355 y ss.

21 Estamos a la espera de analizar la documentación del archivo personal del coleccionista para conseguir esos datos fundamentales. No obstante, la fecha de entrada de La Patronne en la casa del coleccionista Louis Eychenne puede situarse en los años referidos, aunque la fotografía más antigua del cuadro en la casa se data de 1940. Se tomaron fotografías de la obra en 1968 y en 1971, esta última fecha cuando se la preparaba para su envío a la exposición organizada por Nicholas Acquavella (Galería Acquavella) a beneficio del Metropolitan Museum de Nueva York. Sin embargo, la obra no se llegó a prestar debido a que Louis Eychenne sufrió una embolia ese mismo año y se canceló el préstamo. En el año 2008 el cuadro se trasladó a España para ser sometido a estudio y a un análisis riguroso para su limpieza en el IVACOR de la Generalitat Valenciana (ahora integrado en Culturart Generalitat), cuyo resultado fue el Informe técnico de expertizaje científico y restauración de La Patronne de Amedeo Modigliani, a cargo del equipo IVC+r, y de investigadores y restauradores como Greta García Hernández, ofreciendo unas conclusiones científicas, técnicas y estéticas que verifican una concluyente autoría de Modigliani. Desgraciadamente, una serie de circunstancia que superan y quedan al margen de los parámetros de este artículo, impidieron lo que se pretendía por parte de los herederos de Louis Eychenne, que el cuadro se quedase expuesto en Valencia durante dos años, en el Museo de Bellas Artes de San Pío V, junto a los retratos que Foujita y Siqueiros realizaron del coleccionista mexicano. Sobre este incidente vid. Las Provincias, sábado 05.03.2011, p. 48, y CUÑAT, Enric M., "La patronne y los patronos" en <http://www.levante-emv.com/opinion/2011/03/06/patronne-patronos/788097.html >[5/2/13], entre otros ecos sobre el rechazo al cuadro.

22 GARCÍA ORIA, F. y SOTO CABA, V., "Entre Francia y México...», pp. 370-371. 
Entre las incógnitas que plantea el cuadro destacan el cuándo y el dónde se realizó. El colorido usado puede dar pistas en torno a estas cuestiones, ya que una vez restaurado se consiguió devolver su colorido original, eliminando capas de barnices que amarronaban y oscurecían la superficie ${ }^{23}$, consiguiendo desvelar el blanco de gran parte del cuadro y lo que suponemos sería un vano o una ventana. Resulta de enorme interés la aparición del blanco, pues nos encontraríamos en el punto de inflexión de la evolución del pintor con respecto a la luz y al color, un hecho que se produce cuando se traslada a Niza en la primavera de 1918. Como a muchos otros artistas anteriores, la fuerte luz del Midi le impulsó a cambiar de paleta, a aclarar el colorido, a elegir tonos más cálidos y contrastados, a eliminar la contención cromática. Sin embargo, la transformación de la paleta pudo suceder con anterioridad, cuando Modigliani y su compañera Jeanne Hébuterne decidieron irse a vivir juntos, apenas unos meses después de conocerse, a un pequeño piso en la 8 rue de La Grand Chaumière, en realidad un par de habitaciones, una suerte de estudio vecino al de Ortiz de Zárate. Los Zborowski les ayudaron a amueblarlo y el mismo Modigliani pintó las paredes de color naranja, rojo y amarillo, para que sirvieran de fondo a sus modelos ${ }^{24}$, quizá en un intento de revitalizar su colorido, y algunos fondos ocres y rojizos de sus desnudos de 1916 y 1917 lo confirman. Igualmente pintó una ventana y sus cristales de blanco y sus marcos en verde, que bien pueden señalar el plano del fondo de La Patronne. Resulta difícil indagar en la retratada. En francés la patronne puede referirse a la dueña del piso o el inmueble, o a la hija del dueño. Es menos probable que aluda a la portera de la casa, o a su hija, aunque el término se utiliza en ocasiones como sinónimo de concierge. Los apuros y aprietos por los que pasaron el pintor y su pareja obligaron, en plenas estrecheces de la guerra, a realizar y ofrecer retratos a cambio de algún alquiler $y$, en este sentido, es posible que este sea el caso del cuadro y que su realización se date en los años de 1916-1917.

Pero es el retrato de una jovencita, la hija de una patronne, que concuerda con el que realizó Foujita en un lienzo que pertenece a una colección particular ${ }^{25}$ [9]. Ambos retrataron a la misma niña y todo parece indicar que fue en uno de los muchos momentos en que ambos artistas coincidieron, en París en innumerables

23 Véase GARCÍA HERNÁNDEZ, Greta, Estudio de la estructura estratigrafica y posterior limpieza de una obra pictórica: retrato de mujer atribuido a Amedeo Modigliani. Aplicación de los nuevos sistemas de limpieza para la eliminación de las capas de barniz oxidado. (Trabajo fin de Máster Oficial en Conservación y Restauración de Bienes Culturales) Universidad Politécnica de Valencia, UPV, 2008.

24 Este hecho está detallado en LOTTMAN, Herbert, Amedeo Modigliani: príncipe de Montparnasse. Barcelona, Galería Miquel Alzueta, 2008.

25 Un óleo sobre lienzo de 33 × 24 cm; BUISSON, Sylvie lo data hacia 1916-1917; cfr. Léonard Tsuguharu Foujita, ob. cit. 


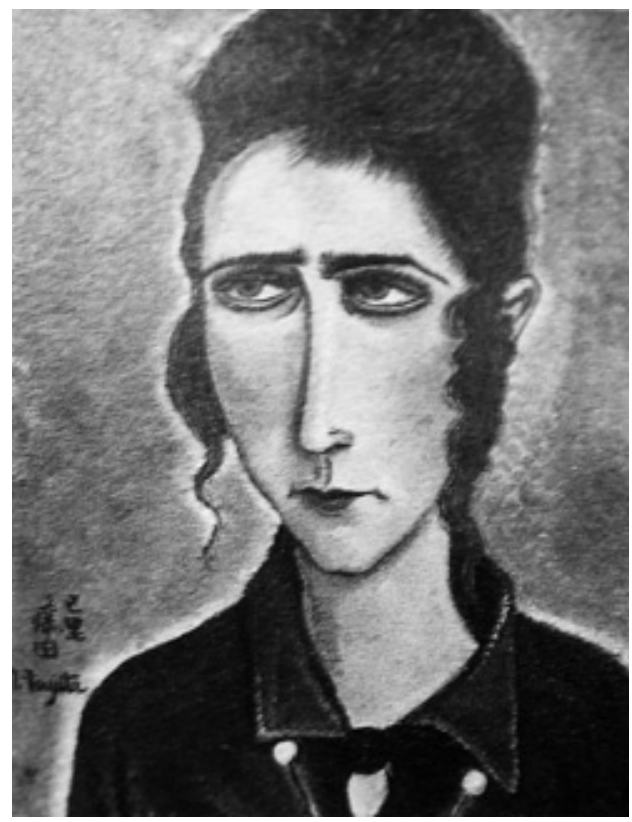

9. Joven de T. Foujita,

c. 1916-1919.

Colección Particular

ocasiones, pero también en el sur de Francia, cuando en 1918, en plena guerra, Leopold Zborowski organizó un viaje a la Costa Azul, con el objetivo de huir de las penurias de la capital gala y de vender cuadros a los turistas ricos, llevándose a sus amigos pintores; y allí, al Midi, fueron Foujita y la entonces su segunda mujer, Fernande Barrey, Soutine, Modigliani y su compañera Jeanne Hébuterne. Los objetivos no se cumplieron, no se vendieron los cuadros esperados, pero sí se produjo una intensa producción en el caso de Modigliani, cuya obra evolucionó hacia un cromatismo más cálido y luminoso ${ }^{26}$. El grupo acabo subsistiendo del dinero que Foujita había ganado en sus exposiciones parisinas, pero cuando se acabó, los cuadros y dibujos se convirtieron en moneda de cambio, un trueque que finalmente no gustó a la patrona del alojamiento y negándose a recibir arte a cambio de deuda decidió requisar el equipaje de los artistas. Tanto Foujita como Modigliani retrataron a la hija de la patronne; aunque es difícil determinar si los cuadros fueron un canje o requisados, acabaron siendo Foujita y Zborowski los encargados de recuperarlos, y pudiera ser que el pintor japonés adquiriese La Patronne a Modigliani como compensación de la inversión que tuvo que asumir. 
10. Madelaine Lequeu. Fotografía, dedicada a Louis Eychenne en México, 1933: «Pour Eychenne, mon meilleur souvenir. Madelaine». Colección

Louis Eychenne, México D.F.

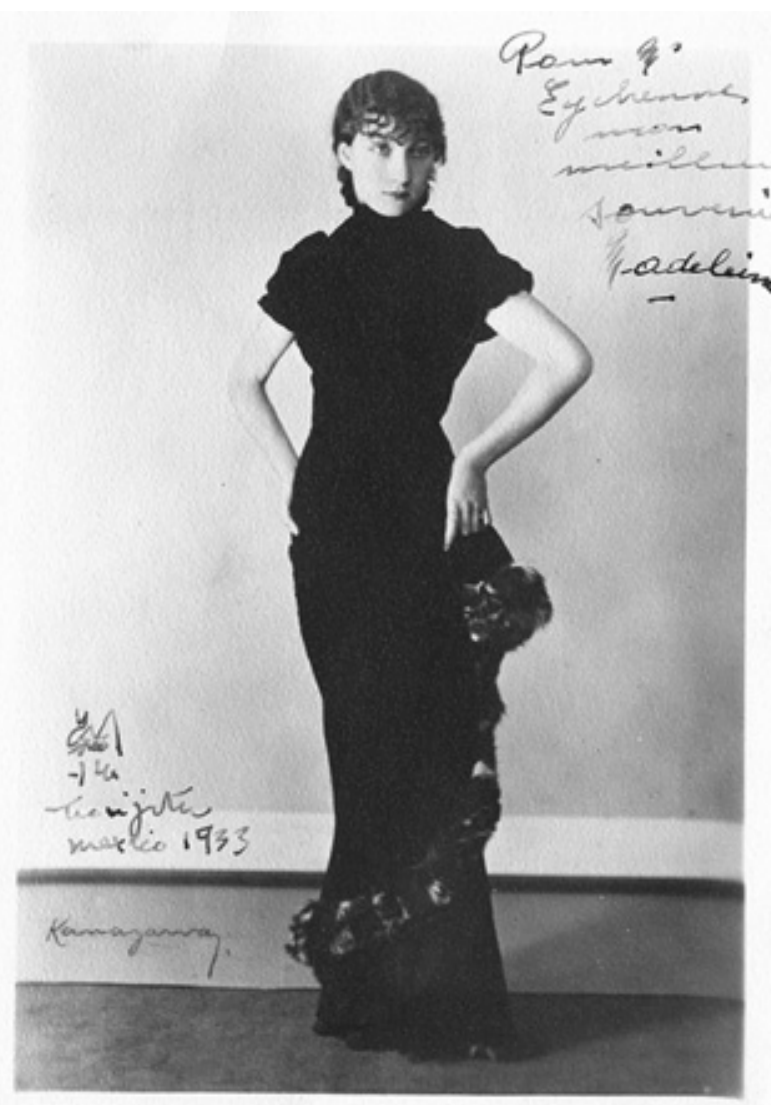

Por otro lado, y entre las numerosas anécdotas que los biógrafos y estudiosos han vertido sobre el artista italiano, hay una que puede relacionarse con el cuadro. A comienzos de 1919, encontrándose en Niza ${ }^{27}$, le roban al pintor su billetera con seiscientos francos, hecho que le impidió regresar a París y que le obliga a pintar apresuradamente retratos, no sobre lienzos, sino sobre cartón, con una gama muy limitada de colores, con una factura muy rápida y a base de pinceladas amplias y ligeras. Se trata de retratos que enviaba a París, en concreto a Zborowski, su mentor y mecenas desde 1916, quien a su vez le remitía dinero por cada obra recibida, una mediación esencial pues gracias a este polaco

27 KRYSTOF, Doris, recalca que apenas existen documentos escritos sobre el año que pasó en el sur de Francia, pero que es muy probable que en un primer momento se alojara en un hotel de Cagnes-sur-Mer para trasladarse más tarde a Niza, Modigliani, Colonia, Taschen, 1916, p. 82. 
sus cuadros se expusieron en Londres. En el sur de Francia Modigliani retomó el contacto con Paul Guillaume, con quien coincidió en Niza, otro intelectual que funcionó de marchante para el pintor italiano, propietario de una galería en la Rue du Faubourg-St.-Honoré, que le compraba y vendía su obra y lo incluía en numerosas exposiciones colectivas de otros artistas entonces desconocidos. Muchos de los cuadros del italiano pasaron por sus manos.

Sin embargo, La Patronne no revela ser un retrato apresurado, ni plantea una factura rápida; sí es cierto que puede verse cierta limitación en los colores para la fecha, pero tampoco refleja la riqueza cromática que tienen los cuadros de 1919. Más bien debería indicarse que continúa trabajando con una economía cromática extremada, solo alterada por la "ventana» blanca, y dentro del estilo seguido desde 1916.

Es difícil, pues, determinar el dónde y el cuándo, pero lo que está claro es que su destino estaba en México, y en los años treinta según el sello de exportación ${ }^{28}$, aunque el trayecto hasta allí, como muchos otras obras de arte que circularon entre los avatares del periodo de entreguerras europeo, está plagada de escalas e hipótesis. Evidentemente pasó desapercibida y no se incluyó en los catálogos que los expertos elaboraron desde mediados de siglo y que, como el de Ambrogio Ceroni ${ }^{29}$, están considerados como piedra angular de los estudios sobre el artista. $Y$ es que la mayoría de los expertos han trabajado más según las necesidades comerciales del momento que en el verdadero estudio de la evolución y catalogación del artista. Sin embargo, hay una importante obra académica y fuente crítica que puede ofrecer una pista sobre el cuadro, los estudios de Arthur Pfannstiel quien publicó en 1929 una visión de la vida de Modigliani año a año ${ }^{30}$ y un catálogo conocido como présumé, en el que menciona en la página 35 una obra que corresponde a las medidas de La Patronne, a la datación y a la procedencia, así como al tema de una "joven pelirroja». Se trata de un trabajo de $1929^{31}$, y asegura la procedencia original de Leopold Zborowski y, posteriormente, su paso por la Galería Bernheim-Jeune. En la segunda edición de Pfannstiel, aparecida en 1956, un catálogo de casi 300 obras, con ilustraciones de casi la mitad, alguna en color, ofrece indicaciones muy precisas sobre la perte-

28 El tipo de sello de salida de la aduana francesa, en el reverso del soporte (Douane Centrale/Exportation/Paris), delata que el cuadro sale de Francia antes de 1936, pues a partir de esta fecha la marca de la impresión del tampón cambia.

29 CERONI, Ambrogio, Tout l'oeuvre Peint de Modiglian, París, Flammarion, 1972. Sus catálogos se remontan a 1952 y están considerados como los más completos, sobre todo el último, de 1972.

30 PFANNSTIEL, Arthur, Modigliani, París, Seheur, 1929.

31 PFANNSTIEL, Arthur, Modigliani. Catalogue présumé, París, 1929. 
11. Madeline Lequeu. Fotografía dedicada, México, 1933: «Pour mon ami Louis ma sincère amitié. Mad Fojita». Colección Louis Eychenne, México D.F.

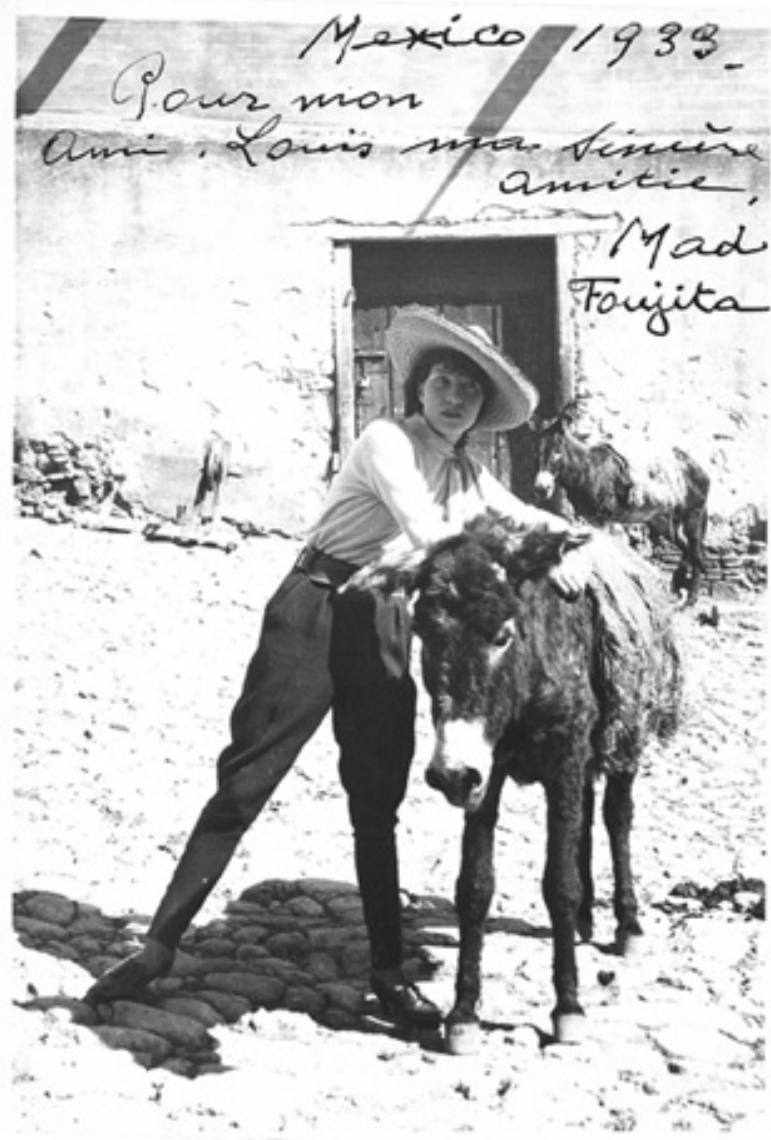

nencia originaria y la colección a la que correspondía los cuadros de Modigliani, así como las publicaciones en las que había figurado y las exposiciones en las que había formado parte ${ }^{32}$. Pero del cuadro de la «joven pelirroja», que aparece con la referencia número 210 , no da aclaraciones ni aporta fotografía, pero hay que tener en cuenta que para esas fechas la obra ya estaba en manos de Louis Eychenne en México.

Por otro lado, hay que incluir un hecho anterior: la estrecha colaboración de Pfannsteil con la Gestapo provocó que sus archivos fueran requisados al final de la guerra y el material de importancia acabó en paradero desconocido.

32 PFANNSTIEL, Arthur, Modigliani et son oeuvre, Étude critique et catalogue raisonné, París, Bibliothèque des Arts, 1956. 
Varias décadas después de su muerte, la parte recobrada de sus archivos acabó en manos de su hijo, y tras ventas y cambios diversos, se integró en los Arhives Legales Paris/Roma de Modigliani dirigidos por Christian Parissot, dando lugar a una total manipulación que desvirtuó el legado y el estudio de Pfannstiel.

Foujita y Madelaine Ilegaron a finales de noviembre de 1933 a Japón. Mientras que él expondrá sus dibujos americanos en 1934, ella regresa a París, pero estará pocos meses, pues volverá con Foujita a finales de este año. La amistad con el mexicano perduró y se sucedieron cartas e intercambios de dibujos del pintor y de fotografías por parte de Mady. Louis les enviaba objetos de artesanía mexicana para el denominado "taller mexicano" de la casa de Tokyo. Un lote de dibujos recibidos le sirvió al coleccionista para organizar otra exposición con cuarenta dibujos y acuarelas de desnudos y temas mexicanos, que tuvo lugar del 2 al 31 de julio, también en el edificio de la Nacional ${ }^{33}$. Muchos de ellos se quedaron en manos de Louis y le sirvieron, durante la década de los años cuarenta, para intercambiar obras a través de su marchante predilecto, el galerista Nicholas Acquavella de Nueva York ${ }^{34}$. Pero para esos años, la amistad entre mecenas y pintor se estaba enfriando. Mady había dejado de ser un nexo, pues había aparecido ahogada en el estanque de su casa, en junio de 1936, víctima de una sobredosis, mientras que Foujita iniciaba uno de los capítulos más polémicos de su trayectoria artística al servicio de la propaganda y el ejército japonés durante la II Guerra Mundial. Fue obligado a glorificar la guerra para recuperar el respeto de sus compatriotas y prolongó el augurio que un crítico le dedicó en los años veinte al calificarle de un hombre que "pasaba por un pintor francés a los ojos de los japoneses y por un puro japonés a los occidentales» ${ }^{35}$. Tuvo que componer inmensos lienzos para el ejército imperial, ajenos a su estilo y a su comedido formato, pero fue una empresa que pudo llevar a cabo gracias a su estancia en México, a su relación con los grandes muralistas y al interés y conocimiento que mostró por la pintura mural, coincidiendo sin duda con otro artista japonés que formó parte de aquel renacimiento plástico, de

33 También en esta ocasión se editó un díptico, Exposición de dibujos con temas mexicanos y desnudos por Foujita, 2 al 31 de julio de 1934. Entre las 16 y las 20 horas. Central de Publicaciones, S.A. Edificio La Nacional, México D.F. Dentro se mencionan los títulos de 44 dibujos.

34 Así lo demuestran las numerosas facturas que se conservan en el archivo de los herederos del coleccionista.

35 SILVER, Kenneth E., "Made in Paris...", p. 46. 
aquella eclosión muralista, Timiji Kitagawa, afincado en México desde 1921 a 1936, año en que regresó a Japón ${ }^{36}$.

Cuando murió en Zurich en 1968 la prensa internacional no dudó en ensalzar al artista japonés más famoso del siglo XX. Tampoco los periódicos de México se olvidaron de su estancia en el país, aunque nadie sabía que en el año en que estuvo, Louis Eychenne era el primer coleccionista mexicano en adquirir o conseguir un modigliani. Pero lo que no olvidaron fue que aquella estancia supuso para el pintor japonés una renovada inspiración, la apertura hacia un nuevo universo y la captación de los rasgos psicológicos de los campesinos e indios mexicanos, como escribía el autor de uno de los obituarios: "Foujita supo estilizar una raza, para mostrarnos lo que hay en ella de angustia, de musical, de melancólico, de pasional, de timidez, de idolátrico y de azoro; para ello necesitó vivir, convivir fraternalmente con nuestros pobres indios, con esos compatriotas que pasan las horas en contemplación sobrehumana a los rayos del sol, bajo nuestro cielo límpido". Recordando una antigua entrevista, el autor de la crónica estimaba la dignidad con que el pintor había captando al indio y ponía en boca de Foujita la frase "estudié los rasgos de los indígenas mexicanos con el deleite con que he copiado a los hombres de mi raza" y en palabras de Madelaine "para hacernos sentir ese hieratismo, esa fuerza oculta, esa inconsciencia que existe en los indios mexicanos, se necesita un gran amor, una profunda admiración por la raza. Y con amor, con fervor, Foujita realizó sus dibujos» ${ }^{37}$.

36 Véase KATO, Kauro, "Acercamiento a la influencia del movimiento muralista en el arte contemporáneo de Japón", Crónicas, México, 2008, n. ${ }^{\circ} 13$, pp. 237-255.

37 JIMÉNEZ, Guillermo, "Murió Foujita, el extraordinario y singular pintor japonés», Magazine de El Universal, México, 20 de octubre de 1968, p. 2. 
